Алгебра и анализ

Том. 17 (2005), вып. 3
St. Petersburg Math. J.

Vol. 17 (2006), No. 3, Pages 465-475

S 1061-0022(06)00915-0

Article electronically published on March 9, 2006

\title{
REGULAR SOLUTIONS OF ELLIPTIC BOUNDARY-VALUE PROBLEMS WITH DISCONTINUOUS NONLINEARITIES
}

\author{
M. G. LEPCHINSKII AND V. N. PAVLENKO
}

\begin{abstract}
The existence of stable solutions to elliptic boundary-value problems is studied; stability is understood with respect to perturbations of nonlinearities. By the variational method, it is shown that stable solutions of such problems do exist provided a certain integral measure of closeness for (possibly, discontinuous) nonlinearities is employed. It is shown that problems with discontinuous nonlinearities can serve as idealization of problems with continuous nonlinearities but having narrow regions of ill-controlled variations with respect to the phase variable.
\end{abstract}

We study the stability of solutions of elliptic boundary-value problems with respect to perturbations of the nonlinearity involved in the equation. The nonlinearity may be discontinuous with respect to the phase variable. The sort of stability that we are interested in can be described as follows. We choose a class $\mathfrak{U}$ of admissible nonlinearities and a measure of closeness for its elements, as well as a distance $d$ in the space $V$ of solutions of the boundary-value problem. We say that a solution $u_{0}(x)$ of the boundaryvalue problem is nl-stable if for any positive $\varepsilon$ there is $\delta>0$ such that if a nonlinearity belongs to $\mathfrak{U}$ and is distant by less than $\delta$ from the nonlinearity of the original problem (in the sense of the chosen measure), then the boundary-value problem with this new nonlinearity has at least one solution in the $\varepsilon$-neighborhood of the point $u_{0}(x)$ of the metric space $(V, d)$. We note that we consider the case where the perturbed problem differs from the original one only by a nonlinearity involved in the equation.

For a bounded domain $\Omega$, the existence of nl-stable solutions for the Dirichlet problem

$$
\begin{aligned}
\Delta u+f(x, u) & =0, \quad x \in \Omega, \\
\left.u\right|_{\partial \Omega} & =0
\end{aligned}
$$

with nonlinearity $f(x, u)$ discontinuous with respect to $u$ was investigated in [1]. In that paper, the class $\mathfrak{U}$ consisted of superpositionally measurable bounded functions satisfying the one-sided Lipschitz condition with one and the same constant for the entire class $\mathfrak{U}$, while the measure of closeness between the elements of $\mathfrak{U}$ was defined in terms of the Hausdorff distance between the corresponding graphs; the metric in $L_{\infty}(\Omega)$ was used as the distance in the space of solutions.

In this paper, the set of admissible nonlinearities $\mathfrak{U}$ is wider, and we consider integral closeness measures, which seems more natural for the problems under study. Note that proximity in an integral sense allows large deviations of nonlinearities from each other (even though only on a set of relatively small measure). The latter feature does not occur for the Hausdorff distance between graphs.

For a sufficiently wide class of elliptic boundary-value problems with discontinuous nonlinearities, we use the variational method to prove the existence of nl-stable solutions

2000 Mathematics Subject Classification. Primary 35J65, 35J50.

Key words and phrases. Elliptic boundary-value problems, discontinuous nonlinearities, strong solutions, nl-stable solutions. 
$u$ with an additional property. Namely, for almost every $x$ in the domain where the equation is considered, the value $u(x)$ is a continuity point (with respect to the phase variable) for the nonlinearity involved in the equation.

As in [2], the nl-stable solutions possessing the additional property mentioned above will be called regular solutions. We establish a result (see Theorem 4) that describes a relationship between the closeness measures on $\mathfrak{U}$ used in [1] and in this paper. For the class of nonlinearities treated in that theorem, the existence result proved in the present paper includes the corresponding result in 1 .

Often, a model with discontinuous nonlinearity arises as idealization of a problem with a nonlinearity that is continuous but may change rapidly as the phase variable runs through some narrow regions. In [3, it was asked to what extent the solutions of an ideal model with discontinuous nonlinearity are close to those of an approximating problem with continuous nonlinearity. The first results in this direction were obtained in [4]. In that paper, the approximating nonlinearity differs from the discontinuous one only near the surface of discontinuity with respect to the phase variable. Here we generalize the results of [4] by using an integral measure of closeness.

\section{Formulation of THE MAIN RESUlts}

Let $\Omega$ be a bounded domain in $\mathbb{R}^{n}(n>2)$ with boundary $\Gamma$ of class $C^{2, \alpha}, \alpha \in(0,1)$, and let

$$
L u(x) \equiv-\sum_{i, j=1}^{n}\left(a_{i j} u_{x_{i}}\right)_{x_{j}}+c(x) u(x)
$$

be a uniformly elliptic differential operator on $\bar{\Omega}$ with coefficients $a_{i j} \in C^{1, \alpha}(\bar{\Omega}), a_{i j}(x)=$ $a_{j i}(x)$ on $\bar{\Omega}, c \in C^{0, \alpha}(\bar{\Omega})$.

We consider the boundary-value problem

$$
\begin{aligned}
L u(x)+g_{0}(x, u(x)) & =0, \quad x \in \Omega, \\
\left.B u\right|_{\Gamma} & =0,
\end{aligned}
$$

where (1.2) is one of the following basic boundary conditions:

the Dirichlet condition if $B u=u$;

the Neumann condition if $B u=\frac{\partial u}{\partial n_{L}} \equiv \sum_{i, j=1}^{n} a_{i j}(x) u_{x_{i}} \cos \left(n, x_{j}\right)$, where the coefficients $\cos \left(n, x_{j}\right)$ are the direction cosines of the outward normal $n$ to the boundary $\Gamma$;

the third boundary condition if $B u=\frac{\partial u}{\partial n_{L}}+\sigma(x) u(x)$, where $\sigma \in C^{1, \alpha}(\Gamma)$ is a nontrivial and nonnegative function on $\Gamma$.

The nonlinearity $g_{0}(x, u)$ satisfies the following conditions $(*)$ :

$* 1)$ the function $g_{0}: \Omega \times \mathbb{R} \rightarrow \mathbb{R}$ is Borel $(\bmod 0)$ [5], which means the existence of a set $l \subset \Omega \times \mathbb{R}$ whose projection to $\Omega$ has zero measure, together with a Borel function on $\Omega \times \mathbb{R}$ that coincides with $g_{0}(x, u)$ on $(\Omega \times \mathbb{R}) \backslash l$;

$* 2$ ) for almost every $x \in \Omega$, the section $g_{0}(x, \cdot)$ has only first kind discontinuities on $\mathbb{R}$, and for any $u \in \mathbb{R}$ we have $g_{0}(x, u) \in\left[g_{-}(x, u), g_{+}(x, u)\right]$, where $g_{-}(x, u)=$ $\liminf _{s \rightarrow u} g_{0}(x, s), g_{+}(x, u)=\limsup _{s \rightarrow u} g_{0}(x, s)$;

$* 3)$ there exists $a \in L_{q}(\Omega), q>n$, such that the inequality

$$
\left|g_{0}(x, u)\right| \leq a(x), \quad u \in \mathbb{R},
$$

is fulfilled for almost all $x \in \Omega$.

We remark that the inequality $q>n$ guarantees that the embedding of the Sobolev space $W_{q}^{2}(\Omega)$ in $C^{1}(\bar{\Omega})$ is compact, which will be used several times. 
Next, we denote by $X$ either the Sobolev spaces $H_{\circ}^{1}(\Omega)$ if $B u=u$, or $H^{1}(\Omega)$ in the case of the Neumann condition or the third boundary condition; $(\cdot, \cdot)$ and $\|\cdot\|$ are the scalar product and the norm in $X$, respectively.

We define a linear bounded operator $\mathcal{L}$ on $X$ as follows:

$$
(\mathcal{L} u, v)=\sum_{i, j=1}^{n} \int_{\Omega} a_{i j}(x) u_{x_{i}} v_{x_{j}} d x+\int_{\Omega} c(x) u(x) v(x) d x+\int_{\Gamma} \sigma(s) u(s) v(s) d s
$$

for any $u, v \in X$, where $\sigma(x) \equiv 0$ for the Dirichlet and Neumann boundary conditions. It is assumed that the operator $\mathcal{L}$ is coercive, i.e., there is a constant $C>0$ such that

$$
(\mathcal{L} u, u) \geq C\|u\|^{2}, \quad u \in X .
$$

It can be shown that $\mathcal{L}$ is coercive if it is nonnegative and has degenerate kernel, in particular, if $c(x)$ is nonnegative on $\Omega$ and, in the case of the Neumann condition, $c(x) \not \equiv 0$ on $\Omega$.

Definition 1. By a strong solution of problem (1.1)-1.2) we mean a function $u \in$ $W_{q}^{2}(\Omega), q \geq 1$, that satisfies equation (1.1) for almost all $x \in \Omega$ and has zero trace $B u(x)$ on the boundary $\Gamma$ of the domain $\Omega$.

Definition 2 [2]. A strong solution $u(x)$ of problem (1.1)-(1.2) is said to be semiregular if for almost every $x \in \Omega$, the value $u(x)$ is a continuity point of $g_{0}(x, \cdot)$.

Definition 3. We say that the A-condition is fulfilled for equation (1.1) if there is an at most countable family of surfaces

$$
\left\{S_{i}, i \in I\right\}, \quad S_{i}=\left\{(x, u) \in \mathbb{R}^{n+1} \mid u=\varphi_{i}(x), x \in \Omega\right\}, \varphi_{i} \in W_{1, \mathrm{loc}}^{2}(\Omega),
$$

such that for almost all $x \in \Omega$ the inequality $g_{0}(x, u-)<g_{0}(x, u+)$ implies the existence of $i \in I$ for which $u=\varphi_{i}(x)$ and

$$
0 \notin\left[L \varphi_{i}(x)+g_{0}\left(x, \varphi_{i}(x)-\right), L \varphi_{i}(x)+g_{0}\left(x, \varphi_{i}(x)+\right)\right] .
$$

For any solution $u(x)$ of the boundary-value problem, the A-condition forbids the situation where for almost all $x$ the value $u(x)$ lies on the discontinuity surfaces of the nonlinearity $g_{0}$ at points of the so-called "jumps" with respect to the phase variable (i.e., at points where $\left.g_{0}(x, u-)<g_{0}(x, u+)\right)$.

Remark 1. If for almost all $x \in \Omega$ the inequality $g_{0}(x, u-) \geq g_{0}(x, u+)$ is fulfilled for every $u \in \mathbb{R}$ (every discontinuity with respect to the phase variable $u$ is a "drop"), then the A-condition for equation (1.1) is fulfilled.

We shall need the following result.

Theorem 1. Assume that the function $g_{0}(x, u)$ occurring in (1.1) satisfies condition (*), and that $u_{0}(x)$ is a local minimum point of the functional

$$
J(u)=2^{-1} \cdot(\mathcal{L} u, u)+\int_{\Omega} d x \int_{0}^{u(x)} g_{0}(x, s) d s
$$

on $X$. Then $u_{0}(x) \in W_{q}^{2}(\Omega)$ satisfies (1.2), and for almost all $x \in \Omega$ we have

$$
-L u_{0}(x) \in\left[g_{-}\left(x, u_{0}(x)\right), g_{+}\left(x, u_{0}(x)\right)\right] \text {. }
$$

In addition, if the A-condition is fulfilled for equation (1.1), then $u_{0}(x)$ is a semiregular solution of problem (1.1) -(1.2).

The proof of Theorem 1 repeats literally the corresponding arguments in the proof of Theorems 1.3 and 1.4 in [6.

The norm in the Lebesgue space $L_{s}(\Omega)$ will be denoted by $\|\cdot\|_{s}(1 \leq s \leq \infty)$.

We introduce two definitions of an nl-stable solution of problem (1.1) (1.2). 
Definition 4. A strong solution $u_{0} \in W_{q}^{2}(\Omega)(q>n)$ of problem (1.1)-(1.2) is said to be nl-stable if for arbitrary $\varepsilon>0$ there exists $\delta>0$ such that the problem

$$
\begin{aligned}
L u(x)+g(x, u(x)) & =0, \quad x \in \Omega, \\
\left.B u\right|_{\Gamma} & =0
\end{aligned}
$$

has at least one semiregular solution in the $\varepsilon$-neighborhood of $u_{0}(x)$ in the space $C^{1}(\bar{\Omega})$ provided that the following conditions are fulfilled: $g(x, u)$ satisfies $(*)$ with the same function $a \in L_{q}(\Omega)$ that occurs in estimate (1.3) for $g_{0}(x, u)$; the inequality

$$
\begin{aligned}
R(g, T) \equiv & \left\|g-g_{0}\right\|_{L_{1}(\Omega \times(-T, T))} \\
& +\left(\left\|u_{0}\right\|_{2 n /(n-2)}+h_{n} \cdot \varepsilon\right)^{\frac{2 n}{p(n-2)}} \cdot\|a\|_{q} \cdot T^{-\frac{1}{n-2}(n+2-2 n / q)}<\delta
\end{aligned}
$$

is true for some $T \geq\left\|u_{0}\right\|_{\infty}\left(h_{n}\right.$ is the norm of the embedding operator from $H^{1}(\Omega)$ into $\left.L_{2 n /(n-2)}(\Omega), p=q /(q-1)\right)$; and the A-condition is fulfilled for equation (1.5).

Definition 5. A strong solution $u_{0} \in W_{q}^{2}(\Omega)(q>n)$ of problem (1.1)-(1.2) is said to be $n l$-stable if for any $\varepsilon>0$ there is $\delta>0$ such that problem (1.5) (1.6) has at least one semiregular solution in the $\varepsilon$-neighborhood of $u_{0}(x)$ in the space $C^{1}(\bar{\Omega})$ provided that the following conditions are fulfilled: $g(x, u)$ satisfies $(*)$ with the same function $a \in L_{q}(\Omega)$ that occurs in estimate (1.3) for $g_{0}(x, u)$; we have

$$
\left\|g-g_{0}\right\|_{L_{1}(\Omega \times \mathbb{R})}<\delta
$$

and the A-condition is satisfied for equation (1.5).

Proposition 1. Let $u_{0}$ be an nl-stable solution of problem (1.1)-(1.2) in the sense of Definition 4. Then so it is in the sense of Definition 5.

Indeed, let $u_{0}$ be an nl-stable solution of problem (1.1)-(1.2) in the sense of Definition 4. Fix $\varepsilon>0$ and choose $\delta>0$ as in Definition 4. Suppose the nonlinearity $g$ satisfies the requirements of Definition 5 and $\left\|g-g_{0}\right\|_{L_{1}(\Omega \times \mathbb{R})}<\delta$. We find a sufficiently large $T$ such that $R(g, T)<\delta$. This can be done because $\left\|g-g_{0}\right\|_{L_{1}(\Omega \times(-T, T))} \leq$

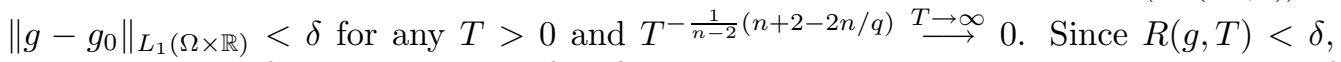
the nl-stability of $u_{0}$ in the sense of Definition 4 implies that in the $\varepsilon$-neighborhood of $u_{0}$ in the space $C^{1}(\bar{\Omega})$ there exists a semiregular solution of the approximating problem (1.5) -(1.6) .

Remark 2. We note that Definition 5 imposes stronger restrictions on the nonlinearity of an approximating problem, whereas Definition 4 requires that the nonlinearities of the initial and approximating problems be close only on the set $\Omega \times(-T, T)$. For this reason, in the sequel we shall use Definition 4.

Definition 6 (see [2]). By a regular solution of problem (1.1)-(1.2) we mean a solution of this problem that is nl-stable (in the sense of Definition 4) and semiregular.

We pass to the statement of the main results.

Theorem 2. Suppose that

1) the function $g_{0}(x, u)$ satisfies condition $(*)$;

2) the coercivity condition (1.4) is fulfilled for operator $\mathcal{L}$;

3) equation (1.1) satisfies the A-condition;

4) the function $u_{0} \in X$ is a point of strict local minimum of the functional

$$
J(u)=2^{-1} \cdot(\mathcal{L} u, u)+\int_{\Omega} d x \int_{0}^{u(x)} g_{0}(x, s) d s
$$

on the space $X$. Then $u_{0}$ is a regular solution of problem (1.1)-(1.2). 
Theorem 3. Suppose that the hypotheses 1)-3) of Theorem 2 are fulfilled, and that the set of points of global minimum of the functional $J(u)$ on $X$ is at most countable. Then problem (1.1) -(1.2) has a regular solution.

Theorem 3 will follow immediately from Theorem 2 if we prove the existence of an isolated point of global minimum for the functional $J(u)$ on $X$. Standard arguments imply that the set $\mathfrak{M}$ of points of global minimum for $J(u)$ is nonempty, because the functional $J(u)$ is weakly lower semicontinuous on $X$ and $\lim _{\|u\| \rightarrow+\infty} J(u)=+\infty$ (see [4]). The continuity of $J(u)$ on $X$ implies that the set $\mathfrak{M}$ is closed. If we suppose that every point of $\mathfrak{M}$ is a limit point for this set, we arrive at the conclusion that $\mathfrak{M}$ is uncountable, as is any perfect closed set in a complete metric space. Thus, the set $\mathfrak{M}$ has an isolated point.

Let $D$ be a metric space with metric $d$. The Hausdorff deviation of a set $A \subset D$ from a set $B \subset D$ in the metric space $(D, d)$ is defined by the formula

$$
\beta(A, B)=\sup _{x \in A} \inf _{y \in B} d(x, y) .
$$

A sequence $\left\{A_{m}\right\}$ of subsets of $D$ is said to be $\beta$-convergent to $A \subset D$ if $\beta\left(A_{m}, A\right) \rightarrow 0$ as $m \rightarrow+\infty$.

The relationship between the measures of closeness of nonlinearities used in [1] and in this paper is clear from the following theorem.

Theorem 4. Suppose that

1) $g_{m}: \Omega \times \mathbb{R} \rightarrow \mathbb{R}, m \in \mathbb{N} \cup\{0\}$, are superpositionally measurable and integrable functions on $\Omega_{R}=\Omega \times(-R, R)$ for any $R>0$;

2) there is an $a \in L_{1}(\Omega)$ such that for any $m \in \mathbb{N} \cup\{0\}$ and almost every $x \in \Omega$ we have

$$
\left|g_{m}(x, u)\right| \leq a(x), \quad u \in \mathbb{R}
$$

$3)$ the closure of the set of discontinuities of the function $g_{0}(x, u)$ in $\Omega \times \mathbb{R}$ has zero measure;

4) for any $R>0$, the sequence of graphs of the functions $\left\{g_{m}(x, u), m \in \mathbb{N}\right\}$ over $\Omega_{R}$ $\beta$-converges to the graph of $g_{0}(x, u)$ over $\Omega_{R}$ in $\mathbb{R}^{n+2}$.

Then for any $R>0$ we have

$$
\int_{\Omega} d x \int_{-R}^{R}\left|g_{m}(x, s)-g_{0}(x, s)\right| d s \rightarrow 0
$$

as $m \rightarrow+\infty$.

For problem (1.1)-(1.2), we consider an approximating sequence of boundary-value problems $(k \in \mathbb{N})$ of the form

$$
\begin{aligned}
L u(x)+g_{k}(x, u(x)) & =0, \quad x \in \Omega, \\
\left.B u\right|_{\Gamma} & =0
\end{aligned}
$$

(the approximating problems differ from the original one only by nonlinearities), where the $g_{k}(x, u)$ satisfy condition $(*)$.

For the functional $J(u)$ on $X$ defined by formula (1.8), let $\mathfrak{M}_{0}$ denote the set of points of global minimum, and let $\mathfrak{M}_{k}$ stand for the same set but for the variational functional $J_{k}(u)$ of problem (1.9) -1.10) $\left(J_{k}(u)\right.$ results from $J(u)$ by replacing $g_{0}(x, u)$ with $\left.g_{k}(x, s)\right)$. We note that if the coercivity condition (1.4) is fulfilled for the operator $\mathcal{L}$, then the sets $\mathfrak{M}_{0}$ and $\mathfrak{M}_{k}$ are not empty.

Theorem 5. Suppose that

1) the functions $g_{k}: \Omega \times \mathbb{R} \rightarrow \mathbb{R}, k \in \mathbb{N} \cup\{0\}$, satisfy condition (*) with one and the same function $a \in L_{q}(\Omega), q>n$, in estimate (1.3); 
2) for any $R>0$ we have

$$
\int_{\Omega} d x \int_{-R}^{R}\left|g_{m}(x, s)-g_{0}(x, s)\right| d s \rightarrow 0 \quad \text { as } k \rightarrow+\infty ;
$$

3) the coercivity condition (1.4) is fulfilled for the operator $\mathcal{L}$.

Then the sequence of sets $\mathfrak{M}_{k} \beta$-converges to $\mathfrak{M}_{0}$ in the space $C^{1}(\bar{\Omega})$.

The following statement is an immediate consequence of Theorem 5

Theorem 6. If the hypotheses of Theorem 5 are fulfilled and $\mathfrak{M}_{0}$ consists of a unique point $u_{0}$, then any sequence $\left\{u_{k}\right\}$ with $u_{k} \in \mathfrak{M}_{k}$ converges strongly to $u_{0}$ in the space $C^{1}(\bar{\Omega})$.

Remark 3. If, in addition to the hypotheses 1)-3) of Theorem [5, the A-condition is satisfied for equation (1.9) for $k \in \mathbb{N} \cup\{0\}$, then $\mathfrak{M}_{k}$ is included in the set of semiregular $W_{q}^{2}(\Omega)$-solutions of problem (1.9)-(1.10) $(k \in \mathbb{N})$. In the case where the nonlinearities in the approximating problem (1.9)-(1.10) $(k \in \mathbb{N})$ are Carathéodory, the A-condition is indeed satisfied for equation (1.9).

\section{§2. Proof of the main Results}

2.1. Proof of Theorem 2. From Theorem 1 it follows that $u_{0}$ is a semiregular $W_{q}^{2}(\Omega)$ solution of problem (1.1)-(1.2). We prove that $u_{0}$ is nl-stable in the sense of Definition 4 .

We fix $\varepsilon>0$. Since $u_{0}$ is a point of strict local minimum of the functional $J(u)$ on $X$, there exists $\nu>0$ such that the inequality $0<\left\|u-u_{0}\right\|_{X} \leq \nu$ implies the strict inequality $J(u)>J\left(u_{0}\right)$. Let $\varepsilon_{1}=\min \{\varepsilon, \nu\}$. We prove the existence of $\kappa>0$ for which

$$
\inf _{\left\|u-u_{0}\right\|=\varepsilon_{1}} J(u)>J\left(u_{0}\right)+\kappa .
$$

Suppose the contrary. Then $\inf _{\left\|u-u_{0}\right\|=\varepsilon_{1}} J(u)=J\left(u_{0}\right)$ because $J(u)>J\left(u_{0}\right)$ whenever $\left\|u-u_{0}\right\|=\varepsilon_{1}$. Hence, there exists a sequence $\left\{u_{m}\right\}$ such that $\left\|u_{m}-u_{0}\right\|=\varepsilon_{1}$ and $J\left(u_{m}\right) \rightarrow J\left(u_{0}\right)$. Since the sequence $\left\{u_{m}\right\}$ is bounded in the reflexive space $X$, without loss of generality we may assume that $u_{m} \rightarrow \hat{u}_{0}$ in $X$. It follows that $\left\|\hat{u}_{0}-u_{0}\right\| \leq$ $\liminf _{m \rightarrow \infty}\left\|u_{m}-u_{0}\right\|=\varepsilon_{1}$, and, by the lower weak semicontinuity of the functional $J(u)$ on $X$ (see [7]), we have

$$
\liminf _{m \rightarrow \infty} J\left(u_{m}\right) \geq J\left(\hat{u}_{0}\right) \geq J\left(u_{0}\right) .
$$

We conclude that $J\left(\hat{u}_{0}\right)=J\left(u_{0}\right)$ because $J\left(u_{m}\right) \rightarrow J\left(u_{0}\right)$. This implies that $\hat{u}_{0}=u_{0}$, because $\left\|\hat{u}_{0}-u_{0}\right\| \leq \varepsilon_{1}$ and $J(u)>J\left(u_{0}\right)$ if $0<\left\|u-u_{0}\right\| \leq \varepsilon_{1}$. Thus, $u_{m} \rightarrow u_{0}$, $J\left(u_{m}\right) \rightarrow J\left(u_{0}\right)$, and $\left\|u_{m}-u_{0}\right\|=\varepsilon_{1}$. Furthermore,

$$
\begin{aligned}
\left(\mathcal{L} u_{m}, u_{m}\right) & =\left(\mathcal{L}\left(u_{m}-u_{0}\right), u_{m}-u_{0}\right)+2\left(\mathcal{L}\left(u_{m}-u_{0}\right), u_{0}\right)+\left(\mathcal{L} u_{0}, u_{0}\right) \\
& \geq C \cdot \varepsilon_{1}^{2}+2\left(\mathcal{L}\left(u_{m}-u_{0}\right), u_{0}\right)+\left(\mathcal{L} u_{0}, u_{0}\right),
\end{aligned}
$$

where $C$ is the constant in the coercivity condition (1.4). Taking the limit and using the selfadjointness of $\mathcal{L}$, we obtain

$$
\liminf _{m \rightarrow \infty}\left(\mathcal{L} u_{m}, u_{m}\right) \geq C \cdot \varepsilon_{1}^{2}+\left(\mathcal{L} u_{0}, u_{0}\right) .
$$

Since $X$ is compactly embedded in $L_{2}(\Omega)$, we have $u_{m} \rightarrow u_{0}$ in $L_{2}(\Omega)$. Recalling estimate (1.3) in condition $(*)$, we see that

$$
\int_{\Omega} d x \int_{0}^{u_{m}(x)} g_{0}(x, s) d s \rightarrow \int_{\Omega} d x \int_{0}^{u_{0}(x)} g_{0}(x, s) d s .
$$


Combined with (2.2), this yields

$$
J\left(u_{0}\right)=\liminf _{m \rightarrow \infty} J\left(u_{m}\right) \geq J\left(u_{0}\right)+2^{-1} \cdot C \cdot \varepsilon_{1}^{2} .
$$

This contradiction proves inequality (2.1).

Suppose that the nonlinearity $g(x, u)$ satisfies condition $(*)$ with the same function $a \in L_{q}(\Omega)$ as in estimate (1.3) for $g_{0}$ and that inequality (1.7) is fulfilled with $\delta=\kappa / 2$ and $T \geq\left\|u_{0}\right\|_{\infty}$. Also, suppose that equation (1.5) satisfies the A-condition. We show that in the $\varepsilon$-neighborhood of the point $u_{0}$ of the space $X$ there exists a semiregular solution of problem (1.5)-(1.6). For this, it suffices to show that, on the sphere $S=$ $\left\{u \in X \mid\left\|u-u_{0}\right\|=\varepsilon_{1}\right\}$, the inequality $J_{g}(u) \geq J_{g}\left(u_{0}\right)$ is fulfilled for the functional

$$
J_{g}(u)=2^{-1}(\mathcal{L} u, u)+\int_{\Omega} d x \int_{0}^{u(x)} g(x, s) d s .
$$

This implies immediately that the functional $J_{g}(u)$ possesses a point of local minimum in the $\varepsilon_{1}$-neighborhood of the point $u_{0}$ in the space $X$, and Theorem 1 applies.

By inequality (2.1), we have

$$
\begin{aligned}
J_{g}(u)= & J_{g}(u)-J(u)+J(u) \\
> & \int_{\Omega} d x \int_{0}^{u(x)}\left(g(x, s)-g_{0}(x, s)\right) d s+J\left(u_{0}\right)+\kappa \\
= & \int_{\Omega} d x \int_{0}^{u(x)}\left(g(x, s)-g_{0}(x, s)\right) d s \\
& -\int_{\Omega} d x \int_{0}^{u_{0}(x)}\left(g(x, s)-g_{0}(x, s)\right) d s+J_{g}\left(u_{0}\right)+\kappa \\
\geq & \kappa-\left(\left|\int_{\Omega} d x \int_{0}^{u(x)}\left(g(x, s)-g_{0}(x, s)\right) d s\right|\right. \\
& \left.\quad+\left|\int_{\Omega} d x \int_{0}^{u_{0}(x)}\left(g(x, s)-g_{0}(x, s)\right) d s\right|\right)+J_{g}\left(u_{0}\right)
\end{aligned}
$$

for any point $u \in S$. We estimate the integrals on the right-hand side in (2.3). Since the space $H^{1}(\Omega)$ is continuously embedded in $L_{2 n /(n-2)}(\Omega)$, for any $u \in S$ we have $\left\|u-u_{0}\right\|_{2 n /(n-2)} \leq h_{n} \cdot \varepsilon_{1}$, where $h_{n}$ is the norm of the operator that embeds $H^{1}(\Omega)$ in $L_{2 n /(n-2)}(\Omega)$. This implies the estimate

$$
\|u\|_{2 n /(n-2)}^{2 n /(n-2)} \leq\left(h_{n} \cdot \varepsilon_{1}+\left\|u_{0}\right\|_{2 n /(n-2)}\right)^{2 n /(n-2)}
$$

for $u \in S$.

Let $\Omega_{u, T}=\{x \in \Omega|| u(x) \mid>T\}$. Then

$$
\int_{\Omega}|u|^{2 n /(n-2)} d x \geq \int_{\Omega_{u, T}}|u|^{2 n /(n-2)} d x \geq T^{2 n /(n-2)} \cdot \text { meas } \Omega_{u, T} .
$$

Consequently, for any $u \in S$ we have

$$
\operatorname{meas} \Omega_{u, T} \leq\left(\frac{h_{n} \cdot \varepsilon_{1}+\left\|u_{0}\right\|_{2 n /(n-2)}}{T}\right)^{2 n /(n-2)}=w_{T} .
$$


The first integral in (2.3) is estimated as follows:

$$
\begin{aligned}
& \left|\int_{\Omega} d x \int_{0}^{u(x)}\left(g(x, s)-g_{0}(x, s)\right) d s\right| \\
& \leq\left|\int_{\Omega_{u, T}} d x \int_{0}^{u(x)}\left(g(x, s)-g_{0}(x, s)\right) d s\right| \\
& \quad+\left|\int_{\Omega \backslash \Omega_{u, T}} d x \int_{0}^{u(x)}\left(g(x, s)-g_{0}(x, s)\right) d s\right| \\
& \quad \leq \int_{\Omega_{u, T}} 2 a(x)|u(x)| d x+\int_{\Omega} d x \int_{-T}^{T}\left|g(x, s)-g_{0}(x, s)\right| d s .
\end{aligned}
$$

Next, applying the Hölder inequality twice, for $u \in S$ we obtain

$$
\begin{array}{rl}
\int_{\Omega_{u, T}} & 2 a(x) \cdot|u(x)| d x \leq 2\|u\|_{2 n /(n-2)} \cdot\left(\int_{\Omega_{u, T}} a^{2 n /(n+2)}(x) d x\right)^{\frac{n+2}{2 n}} \\
\leq & 2\left(h_{n} \varepsilon_{1}+\left\|u_{0}\right\|_{2 n /(n-2)}\right) \cdot\left(\int_{\Omega} a^{2 n /(n+2)}(x) \cdot \chi_{\Omega_{u, T}}(x) d x\right)^{\frac{n+2}{2 n}} \\
\leq & 2\left(h_{n} \varepsilon_{1}+\left\|u_{0}\right\|_{2 n /(n-2)}\right) \cdot\|a\|_{q} \cdot\left(\operatorname{meas} \Omega_{u, T}\right)^{\frac{n+2}{2 n}-\frac{1}{q}} \\
\leq & 2\left(h_{n} \varepsilon_{1}+\left\|u_{0}\right\|_{2 n /(n-2)}\right) \cdot\|a\|_{q} \cdot w_{T}^{\frac{n+2}{2 n}-\frac{1}{q}} \\
& =2\|a\|_{q}\left(h_{n} \varepsilon_{1}+\left\|u_{0}\right\|_{2 n /(n-2)}\right)^{\frac{2 n}{p(n-2)}} \cdot T^{-\frac{1}{n-2}}\left(n+2-\frac{2 n}{q}\right)
\end{array}
$$

where $\chi_{\Omega_{u, T}}(x)$ is the indicator function of the set $\Omega_{u, T}$, and $p=q /(q-1)$.

Thus, for $u \in S$,

$$
\begin{aligned}
& \left|\int_{\Omega} d x \int_{0}^{u(x)}\left(g(x, s)-g_{0}(x, s)\right) d s\right| \\
& \quad \leq 2\|a\|_{q}\left(h_{n} \varepsilon_{1}+\left\|u_{0}\right\|_{2 n /(n-2)}\right)^{\frac{2 n}{p(n-2)}} \cdot T^{-\frac{1}{n-2}\left(n+2-\frac{2 n}{q}\right)} \\
& \quad+\left\|g-g_{0}\right\|_{L_{1}(\Omega \times(-T, T))} .
\end{aligned}
$$

We pass to estimation of the second integral on the right in (2.3). Since $T \geq\left\|u_{0}\right\|_{\infty}$, we have

$$
\begin{aligned}
& \left|\int_{\Omega} d x \int_{0}^{u_{0}(x)}\left(g(x, s)-g_{0}(x, s)\right) d s\right| \\
& \quad \leq \int_{\Omega} d x \int_{-T}^{T}\left|g(x, s)-g_{0}(x, s)\right| d s=\left\|g-g_{0}\right\|_{L_{1}(\Omega \times(-T, T))} .
\end{aligned}
$$

As a result, recalling the choice of $\delta$ in (1.7), from (2.3) we obtain

$$
J_{g}(u)>J_{g}\left(u_{0}\right)+\kappa-\kappa=J_{g}\left(u_{0}\right)
$$

for $u \in S$. As has already been mentioned, this implies the existence of a point of local minimum for the functional $J_{g}(u)$ in the $\varepsilon_{1}$-neighborhood of the point $u_{0}$ in $X$.

At the final step, we argue by contradiction to prove that the solution $u_{0}$ of problem (1.1) - (1.2) is nl-stable. Suppose that $u_{0}(x)$ is not an nl-stable solution of problem (1.1)(1.2) in the sense of Definition 4. Then there exists $\varepsilon>0$, a sequence of numbers $\left\{T_{m}\right\}$ with $T_{m} \geq\left\|u_{0}\right\|_{\infty}$, and a sequence of nonlinearities $\left\{g_{m}(x, u)\right\}$ such that, first, $R\left(g_{m}, T_{m}\right)<\frac{1}{m}$; second, condition $(*)$ is fulfilled with the same function $a \in L_{q}(\Omega)$ as 
in estimate (1.3) for $g_{0}(x, u)$; third, equations (1.9) with $k=m$ satisfy the A-condition; and fourth, for any semiregular solution $u_{m}$ of problem (1.9) -1.10) with $k=m$ we have

$$
\left\|u_{m}-u_{0}\right\|_{C^{1}(\bar{\Omega})} \geq \varepsilon \text {. }
$$

The facts verified above show that for any $l \in \mathbb{N}$ there exists $m_{l} \in \mathbb{N}$ and a local minimum point $u_{m_{1}}$ of $J_{g_{m_{l}}}$ such that the sequence $\left\{m_{l}\right\}$ is monotone increasing and $\left\|u_{m_{l}}-u_{0}\right\|<1 / l$. Theorem 1 implies that $u_{m_{l}}$ is a semiregular $W_{q}^{2}(\Omega)$-solution of problem (1.9)-(1.10) with $k=m_{l}$. By estimate (1.3) in condition $(*)$ for $g_{m_{l}}(x, u)$, and by the coercivity condition (1.4), the sequence $\left\{u_{m_{l}}\right\}$ is bounded in $W_{q}^{2}(\Omega)$ (see [8]). Since the space $W_{q}^{2}(\Omega)$ is reflexive, there exists a subsequence $u_{\tilde{m}_{l}}$ converging weakly to some $\tilde{u} \in W_{q}^{2}(\Omega)$. Since $W_{q}^{2}(\Omega)(q>n)$ is compactly embedded in $C^{1}(\bar{\Omega})$, we see that $u_{\tilde{m}_{l}} \rightarrow \tilde{u}$ in $C^{1}(\bar{\Omega})$ and, as a consequence, $u_{\tilde{m}_{l}} \rightarrow \tilde{u}$ in $X$. But $u_{m_{l}} \rightarrow u_{0}$ in $X$, so that $\tilde{u}=u_{0}$. Thus, $u_{\tilde{m}_{l}} \rightarrow u_{0}$ in $C^{1}(\bar{\Omega})$, which contradicts (2.4).

This concludes the proof of Theorem 2 .

2.2. Proof of Theorem 4. We fix $R>0$, choose $\varepsilon>0$, and set $\delta=\varepsilon /\left(1+2\right.$ meas $\left.\Omega_{R}\right)$. Let $F$ denote the set of discontinuity points for $g_{0}(x, u)$, and let $E$ be the union of $\bar{F}$ and the boundary of the cylinder $\Omega_{R}=\Omega \times(-R, R)$. Next, let $E_{\nu}$ be the $\nu$ neighborhood of $E$ in $\mathbb{R}^{n+1}$. For any $\nu>0$, the set $E_{\nu}$ is Lebesgue measurable in $\mathbb{R}^{n+1}$, and $E_{\nu_{1}} \subset E_{\nu_{2}}$ for $\nu_{1}<\nu_{2}$. Therefore, since $E$ is closed, $E$ coincides with $\bigcap_{\nu>0} E_{\nu}$, whence $\lim _{\nu \rightarrow 0+}$ meas $E_{\nu}=$ meas $E=0$ (by assumption, the closure of the set of discontinuity points of $g_{0}(x, u)$ in $\Omega \times \mathbb{R}$ has zero measure). This implies the existence of $\nu_{1}$ such that for any $m \in \mathbb{N}$ we have

$$
\int_{E_{\nu_{1}, R}}\left|g_{m}(x, s)-g_{0}(x, s)\right| d s d x<\delta
$$

where $E_{\nu_{1}, R}=E_{\nu_{1}} \cap \Omega_{R}$ (we have used condition 2 of Theorem 4 and the absolute continuity of the Lebesgue integral). We note that the set $\Omega_{R, \nu} \equiv \Omega_{R} \backslash E_{\nu, R}$ is closed for any $\nu>0$. Consider the restriction of the function $g_{0}(x, u)$ to the set $\Omega_{R, \nu_{1} / 2}$. By the definition of $\Omega_{R, \nu_{1} / 2}$, the function $g_{0}(x, u)$ is continuous on this set, and, by the Cantor theorem, it is uniformly continuous on $\Omega_{R, \nu_{1} / 2}$. Hence, there exists $\nu_{2}>0$ such that if the distance between two points $z_{1}$ and $z_{2}$ in $\Omega_{R, \nu_{1} / 2}$ is less than $\nu_{2}$, then $\left|g_{0}\left(z_{1}\right)-g_{0}\left(z_{2}\right)\right|<\delta$.

Let $\Gamma_{m}, m \in \mathbb{N} \cup\{0\}$, denote the graph of the function $g_{m}(x, u)$ over $\Omega_{R}$. By condition 4) of Theorem 4 , there exists $m_{0} \in \mathbb{N}$ such that

$$
\beta\left(\Gamma_{m}, \Gamma_{0}\right)<\nu_{3} \equiv \min \left\{\nu_{1} / 2, \nu_{2}, \delta\right\}, \quad m>m_{0}
$$

Suppose $z \in \Omega_{R, \nu_{1}}$. The above inequality shows that for any $m>m_{0}$ there exists $z_{m} \in \Omega_{R}$ such that the distance between the points $\left(z, g_{m}(z)\right)$ and $\left(z_{m}, g_{0}\left(z_{m}\right)\right)$ in $\mathbb{R}^{n+2}$ is less than $\nu_{3}$. This implies that the distance between $z$ and $z_{m}$ in $\mathbb{R}^{n+1}$ and also the number $\left|g_{m}(z)-g_{0}\left(z_{m}\right)\right|$ are less than $\nu_{3}$. By the choice of $\nu_{3}$ and since $z \in \Omega_{R, \nu_{1}}$, this shows that $z_{m} \in \Omega_{R, \nu_{1} / 2}$, whence $\left|g_{0}(z)-g_{0}\left(z_{m}\right)\right|<\delta$. As a result, we obtain

$$
\left|g_{m}(z)-g_{0}(z)\right| \leq\left|g_{m}(z)-g_{0}\left(z_{m}\right)\right|+\left|g_{0}\left(z_{m}\right)-g_{0}(z)\right|<\nu_{3}+\delta \leq 2 \delta
$$


for $m>m_{0}$. Note that $z \in \Omega_{R, \nu_{1}}$ and $m>m_{0}$ are arbitrary. Using estimate (2.5), we obtain

$$
\begin{aligned}
\int_{\Omega} d x & \int_{-R}^{R}\left|g_{m}(x, s)-g_{0}(x, s)\right| d s \\
= & \int_{E_{\nu_{1}, R}}\left|g_{m}(x, s)-g_{0}(x, s)\right| d s d x \\
& +\int_{\Omega_{R, \nu_{1}}}\left|g_{m}(x, s)-g_{0}(x, s)\right| d s d x<\delta+2 \text { meas } \Omega_{R} \cdot \delta=\varepsilon
\end{aligned}
$$

for any $m>m_{0}$. Theorem 4 is proved completely.

2.3. Proof of Theorem 5. It suffices to show that, for an arbitrary monotone increasing sequence of natural numbers $\left\{m_{k}\right\}$, any sequence $\left\{u_{k}\right\}$ with $u_{k} \in \mathfrak{M}_{m_{k}}$ contains a subsequence converging in $C^{1}(\bar{\Omega})$ to some element of $\mathfrak{M}_{0}$. Indeed, otherwise there exists $\varepsilon>0$ and a monotone increasing sequence $\left\{m_{k}\right\} \subset \mathbb{N}$ such that $\beta\left(\mathfrak{M}_{m_{k}}, \mathfrak{M}_{0}\right)>\varepsilon$ for any $k \in \mathbb{N}$. It follows that for each $k \in \mathbb{N}$ there exists an element $u_{k} \in \mathfrak{M}_{m_{k}}$ whose distance to $\mathfrak{M}_{0}$ in $C^{1}(\bar{\Omega})$ exceeds $\varepsilon$. Then the sequence $\left\{u_{k}\right\}$ admits no subsequence converging to an element of $\mathfrak{M}_{0}$, a contradiction.

So, let $\left\{m_{k}\right\}$ be a monotone increasing sequence of natural numbers, and let $u_{k} \in \mathfrak{M}_{m_{k}}$, $k \in \mathbb{N}$. By Theorem 1 the function $u_{k} \in W_{q}^{2}(\Omega)$ satisfies the boundary condition (1.10) and is such that

$$
-L u_{k}(x) \in\left[g_{m_{k}-}\left(x, u_{k}(x)\right), g_{m_{k}+}\left(x, u_{k}(x)\right)\right]
$$

for almost all $x \in \Omega$. This and condition 1) of Theorem 5 imply the boundedness of the sequence $\left\{u_{k}\right\}$ in $W_{q}^{2}(\Omega)$ (see [8]). Since $W_{q}^{2}(\Omega)$ is reflexive, the sequence $\left\{u_{k}\right\}$ contains a subsequence weakly convergent in $W_{q}^{2}(\Omega)$. Without loss of generality, we may assume that the sequence $\left\{u_{k}\right\}$ itself is weakly convergent to $\hat{u}$ in $W_{q}^{2}(\Omega)$. Since $q>n$, the space $W_{q}^{2}(\Omega)$ is compactly embedded in $C^{1}(\bar{\Omega})$; hence, $u_{k} \rightarrow \hat{u}$ in $C^{1}(\bar{\Omega})$. This implies the existence of $\hat{R}>0$ such that for any $k \in \mathbb{N}$ and any $x \in \Omega$ we have $\left|u_{k}(x)\right| \leq \hat{R}$. We fix an arbitrary element $u_{0} \in \mathfrak{M}_{0}$ and put

$$
\nu_{k}=\int_{\Omega} d x \int_{-R}^{R}\left|g_{m_{k}}(x, s)-g_{0}(x, s)\right| d s,
$$

where $R=\max \left\{\hat{R},\left\|u_{0}\right\|_{\infty}\right\}$.

If $u \in C^{1}(\bar{\Omega})$ and $|u(x)| \leq R$ in $\Omega$, then

$$
\begin{aligned}
\left|J(u)-J_{m_{k}}(u)\right| & =\left|\int_{\Omega} d x \int_{-R}^{R}\left(g_{0}(x, s)-g_{m_{k}}(x, s)\right) d s\right| \\
& \leq \int_{\Omega} d x \int_{-R}^{R}\left|g_{0}(x, s)-g_{m_{k}}(x, s)\right| d s=\nu_{k} .
\end{aligned}
$$

In particular, for any $k \in \mathbb{N}$ we have

$$
J\left(u_{k}\right) \leq J_{m_{k}}+\nu_{k}
$$

Since $J$ is a continuous functional on $X$ and $u_{k} \rightarrow \hat{u}$ in $X$, it follows that $\lim _{k \rightarrow+\infty} J\left(u_{k}\right)=$ $J(\hat{u})$. By our hypothesis, $\nu_{k} \rightarrow 0$ as $k \rightarrow+\infty$; hence, (2.6) implies $J_{m_{k}}\left(u_{0}\right) \rightarrow J\left(u_{0}\right)$. Finally, since $u_{k} \in \mathfrak{M}_{m_{k}}$, we have $J_{m_{k}}\left(u_{k}\right) \leq J_{m_{k}}\left(u_{0}\right)$. Combining this and estimate (2.7), we obtain

$$
J(\hat{u})=\lim _{k \rightarrow \infty} J\left(u_{k}\right) \leq \liminf _{k \rightarrow \infty} J_{m_{k}}\left(u_{k}\right) \leq \lim _{k \rightarrow \infty} J_{m_{k}}\left(u_{0}\right)=J\left(u_{0}\right) .
$$


Since $u_{0} \in \mathfrak{M}_{0}$, we have $J(\hat{u}) \geq J\left(u_{0}\right)$. Thus, $J(\hat{u})=J\left(u_{0}\right)$ and $\hat{u} \in \mathfrak{M}_{0}$.

Theorem 5 is proved.

\section{REFERENCES}

[1] M. A. Krasnosel'skiur and A. V. Pokrovskiǔ, Regular solutions of elliptic equations with discontinuous nonlinearities, Proceedings of the All-Union Conference on Partial Differential Equations, Dedicated to the 75th Anniversary of I. G. Petrovskiı̌, Moskov. Gos. Univ., Moscow, 1978, pp. 346347. (Russian)

[2] $ـ$ Regular solutions of equations with discontinuous nonlinearities, Dokl. Akad. Nauk SSSR 226 (1976), no. 3, 506-509; English transl., Soviet Math. Dokl. 17 (1976), no. 1, 128-132. MR0637075 (58:30559)

[3] _ Equations with discontinuous nonlinearities, Dokl. Akad. Nauk SSSR 248 (1979), no. 5, 1056-1059; English transl., Soviet Math. Dokl. 20 (1979), no. 5, 1117-1120 (1980). MR0553925 (81c:45010)

[4] V. N. Pavlenko and R. S. Iskakov, Continuous approximations of discontinuous nonlinearities of elliptic-type semilinear equations, Ukrain. Mat. Zh. 51 (1999), no. 2, 224-233; English transl., Ukrainian Math. J. 51 (1999), no. 2, 249-260. MR1718565 (2000j:35057)

[5] M. A. Krasnosel'skiı̌ and A. V. Pokrovskiı̌, Systems with hysteresis, "Nauka", Moscow, 1983. (Russian) MR0742931 (86e:93005)

[6] V. N. Pavlenko and V. V. Vinokur, Resonance boundary value problems for elliptic-type equations with discontinuous nonlinearities, Izv. Vyssh. Uchebn. Zaved. Mat. 2001, no. 5, 43-58; English transl., Russian Math. (Iz. VUZ) 45 (2001), no. 5, 40-55. MR1860657(2002h:35100)

[7] V. N. Pavlenko, Existence theorems for elliptic variational inequalities with quasipotential operators, Differentsial'nye Uravneniya 24 (1988), no. 8, 1397-1402; English transl., Differential Equations 24 (1988), no. 8, 913-916 (1989). MR0964735 (90a:35101)

[8] S. Agmon, A. Douglis, and L. Nirenberg, Estimates near the boundary for solutions of elliptic partial differential equations satisfying general boundary conditions. I, Comm. Pure Appl. Math. 12 (1959), 623-727. MR0125307 (23:A2610)

Numerical Mathematics Department, Chelyabinsk State University, Brat'ev Kashirinykh Street 129, Chelyabinsk 454021, Russia

E-mail address: myth@csu.ru

Numerical Mathematics Department, Chelyabinsk State University, Brat'ev Kashirinykh Street 129, Chelyabinsk 454021, Russia

Received 26/MAY/2005

Translated by I. V. DENISOVA 